overall cross section, and when it is combined with the direct inelastic and the one-step transfer it gives a distribution that agrees very well with the experimental data. The inclusion of the two-step process has thus essentially accounted for the phase difference in the previous comparison without this process.

The full calculation with the one and two step transfer processes as well as the direct inelastic scattering was repeated for some other energies and they were seen to give a very good overall account of the change of the cross section with energy. It is likely that the small discrepancies that remain can be accounted for by higher order transfer processes. The calculations also give the cross sections for elastic scattering, and these agree well with the experimental data.

\section{Flares and spots on dwarf $M$ stars}

\author{
from R. A. E. Fosbury
}

Probably the most common selfluminous objects in the Universe are the dwarf $\mathbf{M}$ stars: stars which have a mass less than a few tenths that of the Sun but a luminosity which is smaller by a considerably larger factor. Their faintness has, until quite recently, resulted in neglect by observers and theoreticians alike. But this property is an advantage in the study of some curious phenomena which take place in their outer atmospheres. A major flare can increase the brightness of one of these little stars by more than five magnitudes (a factor of one hundred) for a period of a few minutes.

The high kinetic temperatures found in the solar chromosphere and corona are thought to result from the dissipation of a mechanical energy flux which has its origin in the hydrogen convection zone beneath the photosphere. The magneto-acoustic wave modes which are allowed to propagate upwards through the temperature minimum between the photosphere and chromosphere travel through regions of decreasing gas density until they are forced to dissipate their energy as heat through some form of shock. By analogy this form of non-radiative heating is believed to operate in all stars which have a significant convection zone near their surface, that is, those stars which have a spectral type later (cooler) than about F5. Another consequence of the outwardly decreasing density in a stellar atmosphere is the importance of any surface magnetic field in controlling the morphology above the height at which the gas and magnetic pressures are approximately equal. It is the resulting structural in- homogeneity which makes the quantitative study of the chromospheres of stars other than the Sun so difficult.

The existence of stellar chromospheres can be inferred from observations of spectral lines whose profiles show that the temperature increases outwards through part of their region of formation. The best known of these are the famous violet $\mathrm{H}$ and $\mathrm{K}$ lines of ionised calcium which can be detected in emission in a very large number of late-type stars. Even more useful chromospheric indicators are the analogous lines of ionised magnesium at $280 \mathrm{~nm}$ which are now being observed from balloon, rocket and satellite. A subset of the dwarf $M$ stars, known as dMe stars, show also the Balmer lines of hydrogen in emission and it is from this subset that are drawn most, if not all, of the known flare stars.

The flare stars have now provided the clearest observational evidence so far of the existence of starspots. Observations of quasi-periodic brightness variations (outside times of flare activity) by Evans and Bopp at Texas and others have been interpreted as a rotational modulation caused by large dark areas drifting across the visible hemisphere. These spots can cover as much as 50 degrees in latitude and longitude but the actual sizes they derive depend to some extent on the temperatures assigned to them. One might naively think that the sizes of such surface inhomogeneities are fixed in some way by the pressure scale height in the atmosphere, the ratio of this quantity to the stellar radius being roughly constant along the main sequence. D. J. Mullan (Astrophys. J., 186, 1059; 1973) however has put forward the hypothesis that sunspots can be considered as convection cells of some type which extend to the bottom of the solar convection zone. In his latest paper (Astrophys. J., 192, 149; 1974) he extends this idea to the flare stars which, with their position lower down the main sequence, have envelope convection zones with a much greater radial extent. Since the ratio of spot diameter to depth has a minimum value of about two in his theory, he predicts that the diameters of spots should be greater in later spectral types. The predicted sizes are in good agreement with observation.

When the convection zone is confined to a thin shell, as it is in the Sun, the dynamo-generated magnetic field is made up primarily of the higher order multipoles. As the convection zone becomes deeper, however, the lower order multipoles come to dominate until in a fully convective star the field should be mainly dipolar. Mullan suggests that this puts a lower limit on the mass, and hence luminosity, of stars which might be expected to show a rotational modulation of brightness. If the field is dipolar with rotational and magnetic axes parallel then the spots would be expected to form symmetrically around the poles.

Mullan computes a model for a spot on the flare star YY Gem and estimates a surface field in the spot of about 20 kilogauss. Such large magnetic fields are probably generated by efficient dynamos driven by fast differential rotation. There is some evidence that tidal effects may be partly responsible for triggering the sudden release of energy contained in a magnetic field, resulting in a stellar flare.

\section{Macrophages eat their way into genetic control of immunity}

\section{from Robert Kerbel}

Macrophages seem to exist solely for the amusement of immunologists, enabling them to re-discover their importance about every five years. This predictable cycle has been upset by recent events, and one could say it's been a good year-or two-for the macrophage.

To begin with, it appears the macrophage has been restored to its position of being the principal aggressor cell in anti-tumour immunity systems (see for example Eccles and Alexander, Nature, 250, 667; 1974) after being temporarily eased off centre stage by the $T$ cell. Then there was the work of Feldmann and colleagues which assigned a role to macrophages in antibody formation beyond the mere digestion and processing of antigen (Feldmann and Nossal, Transplantn. Rev., 13, 3; 1972); these authors reason that the surface of certain types of macrophage is the main site of $T$ and $B$ cell collaboration, by virtue of the highly cytophilic nature of an antigen-specific factor (IgT, as it is called) liberated by activated $\mathrm{T}$ cells, for the surface of macrophages.

But at least these are areas in which macrophages have a I ways been thought to play a part, even if the importance of their role varied from one year to the next. A recent set of papers from the laboratory of J. G. Howard, however, now transports macrophages firmly into an area in which they were never seriously considered previously to have a role-genetic control of the immune response (Howard, Courtenay and Desaymard, Eur. J. Immun., 4, 453; 1974; and Weiner and Bandieri, Eur. J. Immun., 4, 457; 1974). The authors' model involved the use of so-called Biozzi 'high' and 'low' responder mice; these are two lines of mice $(\mathrm{Ab} / \mathrm{H}$ and $\mathrm{Ab} / \mathrm{L}$ ) which were genetically selected 